\title{
O comprometimento do equilíbrio em integrantes da Associação Missioneira de Parkinson de Santo Angelo-
}

RS

\author{
The impairment of balance among the patients of the Associação Missioneira de Parkinson at \\ Santo Angelo-RS
}

\section{Priscila de Lima Ribeiro', Carlos Kemper²}

\begin{abstract}
RESUMO
Objetivo. Verificar o nível de equilíbrio em indivíduos portadores da Doença de Parkinson, integrantes da Associação Missioneira de Parkinson de Santo Ângelo-RS (AMPark). Método. Foi realizada uma pesquisa quase-experimental com delineamento pós facto, com 14 indivíduos divididos em dois grupos, sendo o primeiro composto por indivíduos portadores da Doença de Parkinson (GParkinson N=7) e o segundo pelos seus Cuidadores (GCuidador $\mathrm{N}=7$ ). Os sujeitos foram submetidos ao Teste de Equilíbrio de Berg composto por 14 tarefas que envolvem equilíbrio estático e dinâmico. Resultados. Sujeitos integrantes do GParkinson obtiveram 42,12 pontos e GCuidador 54,43 pontos $(p<0,05)$ que, de acordo com a classificação apresentada por Shumway-Cook \& Woollacott, representam valores significantes $73 \%$ e $7,14 \%$, respectivamente. Conclusão. Este estudo mostrou que há um comprometimento do equilíbrio em portadores da Doença de Parkinson integrantes da AMPark Santo Ângelo-RS.
\end{abstract}

Unitermos. Equilíbrio Musculosquelético, Doença de Parkinson, Quedas.

Citação. Ribeiro PL, Kemper C. O comprometimento do equilíbrio em integrantes da Associação Missioneira de Parkinson de Santo Angelo-RS.

Trabalho realizado na Universidade Regional Integrada do Alto Uruguai e das Missões - Campus de Santo Ângelo. Santo ÂngeloRS, Brasil.

1. 1.Pós Graduanda em Exercício Físico e Treinamento Esportivo pela Universidade Regional Integrada do Alto Uruguai e das Missões Campus de Santo Ângelo; Santo Ângelo - RS, Brasil.

2.

3. 2.Mestre em Atividade Física e Saúde. Professor da Universidade Regional Integrada do Alto Uruguai e das Missões - Campus de Santo Ângelo. Santo Ângelo-RS, Brasil.

\section{SUMMARY}

Objective. To verify the balance in patients with Parkinson Disease of the Associação Missioneira de Parkinson (AMPark) at Santo Ângelo-RS. Method. An experimental research with a post factum outline has been carried out and 14 individuals, divided into two groups: seven patients with Parkinson Disease (GParkinson) and 7 caregivers (GCaregiver). The individuals were submitted to the Berg Equilibrium Test with 14 tasks of static and dynamic balance. Results. The Parkinson Disease patients had scored 42.12 and the caregivers scored 54.43 $(p<0.05)$. According to Shumway-Cook\&Woollacott classification, those scores represent $73 \%$ and $7.14 \%$ respectively in terms of significant values. Conclusion. The research shows that there is balance impairment in Parkinson Disease patient of the AMPark at Santo Angelo-RS.

Keywords. Musculoskeletal Equilibrium, Parkinson Disease, Accidental Falls

Citação. Ribeiro PL, Kemper C.The impairment of balance among the patients of the Associação Missioneira de Parkinson at Santo Angelo-RS
Endereço para correspondência: Priscila de Lima Ribeiro R. do Carmo, 641 CEP98804-280, Santo Ângelo-RS, Brasil. priscila.prikarib@gmail.com

Recebido em: 15/01/2009 Revisado em: 16/01/2009 a 23/08/2009

Aceito em: 24/08/2009

Conflito de interesses: não 


\section{INTRODUÇÃO}

A Doença de Parkinson é uma patologia neurodegenerativa do Sistema Nervoso Central, que ocorre devido a um distúrbio nos gânglios basais, acarretando na perda de células produtoras da dopamina (precursor natural da adrenalina e noradrenalina). A perda dessas células está associada ao envelhecimento natural, porém, apresenta-se mais acelerada nos portadores da Doença de Parkinson'

Esta patologia manifesta-se de forma assintomática e sua evolução varia de acordo com cada indivíduo. Na maioria dos casos, quando os sintomas ficam mais evidentes e a Doença é diagnosticada, cerca de $70 \%$ das células já foram perdidas ${ }^{1}$.

Sendo neurodegenerativa, a Doença de Parkinson apresenta uma deficiência nos sistemas neurológico e neuromuscular, ocasionada por um distúrbio no ganglio basal que gera uma carência na resposta ao estímulo enviado para a medula espinhal, responsável pelo controle da ação muscular. Dessa forma, ocorre uma diminuição da aptidão física do indivíduo ${ }^{2-4}$.

Dentre suas principais manifestações clínicas estão a rigidez muscular, que se manifesta com o aumento da resistência que os músculos oferecem quando um segmento do corpo é deslocado passivamente ${ }^{5}$. A hipocinesia, definida como movimentos de coordenação com amplitude reduzida, inclui a incapacidade de cronometrar a ativação dos músculos, gerando um movimento mais lento (bradicinesia), com baixa intensidade e grande dispêndio energético, provocando fadiga no indivíduo 5 .

Já o tremor, envolve um movimento rítmico do polegar na direção dos dedos, seguido de uma abdução e adução do polegar; podem ocorrer também pronação e supinação alternadas dos pulsos em repouso, o que se torna bem visível quando o indivíduo está com o membro em repouso porem em movimento, este tremor diminui ou desaparece. Por fim, a instabilidade postural se faz presente devido às alterações ocorridas nos sistemas neurológicos e neuromusculares que são responsáveis pela manutenção postural do indivíduo que comprometem a musculatura do tronco, causando assim, uma postura anormal ${ }^{6}$.

Estudos realizados comprovam a influência da prática do exercício físico para o aumento dos níveis de independência do indivíduo idoso ${ }^{2,7}$. No estudo realizado por Braga $^{2}$ foram analisados os benefícios do treinamento resistido na reabilitação da marcha e equilíbrio em portadores de Doença de Parkinson, apresentando resultados significativos relacionados ao tempo de execução dos exercícios e melhora na força dos músculos envolvidos na marcha e equilíbrio. Sendo assim, procura-se verificar o nível de equilíbrio em indivíduos portadores da Doença de Parkinson, frente às condições neurodegenerativas ocasionadas pela patologia.

\section{MÉTODO \\ Amostra}

A amostra deste estudo foi composta por 14 indivíduos voluntários, sendo 7 portadores da Doença de Parkinson (GParkinson) e 7 Cuidadores (GCuidador), pertencentes a Associação Missioneira de Parkinson de Santo Ângelo - RS. Considerase cuidador o indivíduo que acompanha e auxilia diariamente o portador da Doença de Parkinson, destinando-lhe um cuidado especial. Estes indivíduos geralmente são integrantes da própria família do portador.

Esta pesquisa foi aprovada pelo Comitê de Ética da Universidade Regional Integrada do Alto Uruguai e das Missões - Campus de Santo Ângelo, no dia 09/04/2007, com número do protocolo CAAE - 0057.0.282-07.

\section{Procedimento}

Após assinatura dos devidos Termos de Consentimento livre e esclarecido, foi realizado o Teste de Equilíbrio de Berg ${ }^{5}$, constituído por uma bateria de 14 tarefas comuns que envolvem o equilíbrio estático e dinâmico, tais como alcançar, girar, transferir-se, permanecer em pé e levantar-se. Esta bateria de testes é controlada por um orientador, que anotará os pontos obtidos em cada tarefa, bem como controle do cronômetro e supervisão dos indivíduos durante a execução das atividades.

Para cada tarefa solicitada, tem-se uma pontuação específica que vai de zero a quatro pontos, onde, ao final do teste, a soma destes pontos informa o nível de equilíbrio que o indivíduo apresenta. Qualquer alteração nas atividades, como apoiar-se em um suporte externo, receber ajuda do examinador ou ainda se não alcançar o tempo e a distância necessários, a pontuação será reduzida.

A classificação dos resultados foi realizada de acordo com a pontuação apresentada, onde, de 56 a 54 e cada ponto a menos há um aumento de 3 a $4 \%$ no risco de quedas; de 54 a 46 , a alteração de um ponto é associada ao au- 
mento de 6 a $8 \%$ de chances e, abaixo de 36 pontos, o risco de quedas é quase de $100 \%$.

\section{Análise Estatística}

Para a análise dos dados foi realizado o teste " $\mathrm{t}$ " Student para amostras independentes com o nível de significância de $p \leq 0,05$ bem como a análise descritiva dos dados, sendo utilizado o pacote estatístico SPSS for Windows 11.5.

\section{RESULTADOS}

Para verificar a magnitude do comprometimento do equilíbrio em indivíduos portadores da doença de Parkinson foi realizado o Teste de Equilíbrio de Berg, dividido em tarefas que, de acordo com o desempenho do indivíduo, determinam uma pontuação e permitem mensurar o risco de quedas dos indivíduos.

Observou-se que o GParkinson apresentou valores significativos de 42,14 pontos, enquanto que o GCuidador obteve 54,43 pontos ( $p<0,05$; Tabela 1). Na análise das pontuações, pode-se observar o elevado risco de quedas dos portadores da Doença de Parkinson, totalizando $73 \%$, enquanto que aos cuidadores, apenas 7,14\%, mostrando um comprometimento do equilíbrio provocado pela doença.

Tabela 1. Resultados do Teste de Equilíbrio de Berg dos grupos GParkinson e GCuidador, e classificação do Risco de Quedas.

\begin{tabular}{|l|l|l|l|l|}
\hline & \multicolumn{2}{|l|}{ G PARKINSON } & \multicolumn{2}{l|}{ G CUIDADOR } \\
\hline & MÉDIA & DP & MÉDIA & DP \\
\hline $\begin{array}{l}\text { Teste de Berg } \\
\text { (pontos }\end{array}$ & $42,14^{*}$ & 8,60 & 54,43 & 1,51 \\
\hline $\begin{array}{l}\text { Risco de Quedas } \\
\text { (\%) }\end{array}$ & $73,00^{*}$ & 29,75 & 7,14 & 7,75 \\
\hline
\end{tabular}

${ }^{*} p<0,05$

\section{DISCUSSÃO}

No ser humano em geral, a função do controle postural é dar suporte, estabilização e equilíbrio, realizado através da contração muscular, assegurando o equilíbrio durante a execução de atividades dinâmicas ou estáticas ${ }^{8}$. Na ausência de alguns destes fatores, o equilíbrio é comprometido e o risco de quedas aumenta (devido a força da gravidade), pois o equilíbrio é possui grande importância no desenvolvimento das atividades diárias, complexas ou as mais simples possíveis, como caminhada.

O Colégio Americano de Medicina do Esporte (ACSM, 1998) afirma que a instabili- dade postural ocorre quando os sistemas sensorial e motor são afetados, bem como gânglio basal, cerebelo e sistema perceptivo, que interpreta a informação sensorial recebida?.

Para o portador da Doença de Parkinson, o equilíbrio é prejudicado devido ao distúrbio no gânglio basal que gera uma carência na resposta ao estímulo enviado para a medula espinhal, responsável pelo controle da ação muscular. Dessa forma, ocorre uma instabilidade postural, além da perda de força, potência e resistência muscular, limitando a capacidade de execução das AVD's's

As qualidades físicas, voltadas para a saúde, não incluem o equilíbrio como um fator de aptidão, porém ele é considerado uma qualidade física de grande importância. Não existem relatos que a Doença de Parkinson comprometa os órgãos responsáveis pela manutenção do equilíbrio, ocorrendo sim, uma carência na resposta ao estímulo, devido à morte de células produtoras de dopamina. Esta carência ocorre nos gânglios basais que, ao enviar um estímulo para a medula (que controla a ação muscular), provocando uma instabilidade postural e, assim, um aumento no risco de quedas ${ }^{2-4}$.

O principal objetivo de um treinamento para portadores da Doença de Parkinson é manter as capacidades funcionais do paciente, principalmente motoras, incluindo flexibilidade, equilíbrio, força muscular e coordenação. Um programa de treinamento não retarda a progressão dos sintomas da doença, porém previne complicações secundárias como perda de força muscular, redução na amplitude dos movimentos, hipotensão, osteoporose induzida pela inatividade. Com a melhora destes parâmetros, consequentemente surgirão benefícios ao sistema cardiovascular'.

Dentre os benefícios proporcionados pela prática de exercício físico para portadores da Doença de Parkinson estão a melhora na velocidade e execução dos movimentos, causadas pelo aprimoramento da força muscular e amplitude de movimentos; redução da rigidez muscular; melhora na coordenação muscular, reduzindo o dispêndio de energia durante a execução de atividades. A melhora no desempenho cardiovascular depende da intensidade do exercício realizado, e que o tempo de apoio em uma perna durante a execução da marcha resulta na melhora do equilíbrio'.

Em um estudo de caso, com objetivo de analisar as condições motoras de um portador da Doença de Parkinson, foi constatado que exis- 
tem grandes dificuldades na realização de tarefas diárias como transferências e caminhar, pois o indivíduo apresenta um padrão de atividade muscular diferente, que ativa ambos os lados do corpo. Em situação de instabilidade, os movimentos são executados com menor amplitude e em uma velocidade que o indivíduo tenha segurança. Com isso, surge a capacidade reduzida de coordenar articulações múltiplas. Além disso, foi observado que o tremor acarreta conseqüências na força, interrompendo sua trajetória, estimulando uma força excessiva ao pegar um objeto e dificultando a ação para soltá-lo. Por estes achados de, há uma demonstração clara que a doença realmente compromete o equilíbrio, vindo ao encontro a este estudo que mostrou um alto comprometimento no equilíbrio destes indivíduos ${ }^{5}$.

Para amenizar os efeitos da falta de equilíbrio de indivíduos portadores da Doença de Parkinson, são apresentados benefícios do exercício na reabilitação da marcha e equilíbrio dos portadores da Doença de Parkinson, auxiliando na prática das atividades diárias, independência e reintegração social. Para isto, foi realizado um treinamento durante 13 semanas, contando com 2 sessões semanais de $1 \mathrm{~h} 30 \mathrm{~min}$ cada, e dentre as atividades propostas, estavam a caminhada, fortalecimento muscular, alongamentos. Foram realizados testes pré e pós treinamento, para avaliar a melhora no desempenho do indivíduo. Os testes mostraram que, dentre diversos benefícios oferecidos pelo treinamento, houve um aumento no tônus e músculos envolvidos na marcha e equilíbrio propiciando passadas mais cadenciadas e coordenadas com os membros superiores ${ }^{2}$.

Para reforçar estes achados, foi realizado outro treinamento, sobre as influências no controle do equilíbrio de idosos, desenvolvido em um período de 7 meses, com duas sessões semanais, de $1 \mathrm{~h} 30 \mathrm{~min} /$ sessão. As atividades realizadas foram de reestruturação corporal, capacidades físicas, motoras e habilidades básicas utilizadas diariamente e, para análise, foram realizados testes de campo e de laboratório nos períodos pré e pós treinamento. Os resultados apresentados mostraram um aumento no alcance funcional dos indivíduos, oportunizando uma melhora no controle do equilíbrio e, consequentemente, um aumento nos níveis de independência. Concluindo, então, que a prática do exercício físico contribui para a melhora do equilíbrio comprometido pela doença de Parkinson? ${ }^{7}$.
Outro experimento foi realizado, com objetivo de aprimorar a força muscular, flexibilidade, equilíbrio e função respiratória. Para isto, foram realizados treinamentos de duas sessões semanais, sendo a primeira com ênfase na redução da rigidez, utilizando a resistência da água para realização do trabalho de força. Outra sessão era realizada em um ginásio de esportes, objetivando a melhora dos movimentos de marcha, flexibilidade e coordenação motora. Nos resultados apresentados, o tempo de caminhada reduziu durante o período de treinamento, em virtude das sessões de exercícios os pacientes se sentiam melhor emocionalmente. Houve melhora significativa no desempenho da função motora, realização das atividades diárias, redução na duração e intensidade de discinesia'.

\section{Conclusão}

Com os resultados apresentados no teste, foi possível classificar os indivíduos quanto ao seu risco de quedas, onde pode-se concluir que os indivíduos portadores da Doença de Parkinson, integrantes da AMPark Santo Ângelo-RS, apresentam um comprometimento do equilíbrio em torno de $73 \%$, comparado aos seus Cuidadores, $7,14 \%$, mensurado pelo Teste de Equilíbrio de Berg.

\section{Referências}

1.Reuter I, Engelhardt MK. Doença de Parkinson. In: Lemura LM, Duvillard SPV. Fisiologia do Exercício Clínico - Aplicações e Princípios Fisiológicos. Rio de Janeiro: Gaunabara Koogan, 2006, p214-27.

2.Braga A, Xavier A, Machado POR, Marques MB. Benefícios do Treinamento Resistido na Reabilitação da Marcha e Equilíbrio nos Portadores da Doença de Parkinson. Rev Dig Vida Saúde 2003;2:9.

3.Camargos ACR. O Impacto da Doença de Parkinson na Qualidade de Vida: uma Revisão de Literatura. Rev Bras Fisioter 2004;8:267-72.

4.Goulart FRP, Barbosa CM, Silva CM, Teixeira-Salmela L, Cardoso FO. Impacto de um programa de Atividade Física na Qualidade de Vida de pacientes com Doença de Parkinson. Rev Bras Fisioter 2005;9:49-55.

5.Shumway-Cook A, Woollacott MH. Controle Motor - Teoria e Aplicações Práticas. 2a ed. São Paulo: Editora Manole, 2003, numero total de paginas.

6.Thistlethwaite J. Doença de Parkinson. Rev Neurol Update 2002:15-8.

7.Ramos BMB. Influências de um Programa de Atividade Física no Controle do Equilíbrio de Idosos (monografia). São Paulo: Universidade de São Paulo, 2003, 65p.

8.Rothwell J. Control of Human voluntary movement. 2.ed. London: Chapman\& Hall, 1994, p. 252.

9.ACSM - Posicionamento Oficial do Colégio Americano de Medicina Esportiva - Exercício e Atividade Física para Pessoas Idosas. Rev Bras Med Esp 1999;5:207-11. 